
\title{
25 Research Soure \\ Oral Candidiasis in Patients with Hematological Diseases: Diagnosis Through Clinical and Cytopathological Exams
}

Fernanda Guimarães de Azevedo Pereira

Universidade Federal Fluminense (UFF)

\section{Adrianna Milagres}

Universidade Federal Fluminense (UFF)

Juliana Tristão Werneck

Universidade Federal Fluminense

Letícia Côgo Marques ( $\square$ leticiacogo@hotmail.com )

Universidade Federal Fluminense (UFF)

\section{Bruna Lavinas Sayed Picciani}

Hospital Universitário Antônio Pedro, Universidade Federal Fluminense

Arley Silva Junior

Universidade Federal Fluminense (UFF)

\section{Research Article}

Keywords: Candidiasis, pathology, Microbiology, hematologic diseases, oral manifestations.

Posted Date: November 1st, 2021

DOI: https://doi.org/10.21203/rs.3.rs-1025073/v1

License: (1) This work is licensed under a Creative Commons Attribution 4.0 International License. Read Full License 


\section{Abstract}

Background: Candidiasis is a common opportunistic infection that may interfere with oncologic patients' prognosis, especially those with hematologic diseases. This study is the first to analyse the prevalence of oral candidiasis in onco-hematological patients by physical and oral cytopathological exams.

Methods: This is a cross-sectional and observational study with a retrospective sample composed of participants hospitalized in the hematology clinic, diagnosed with hematologic diseases. All patients were submitted to an oral mucosal exam and scraps from oral mucosa were obtained.

Results: Of the 62 participants, $56.5 \%$ were male, $82.3 \%$ were white, with mean age of 57 years. Lymphoma was the most common hematologic disease (24.2\%). In total, $48.3 \%$ of the sample was diagnosed with oral candidiasis. Of these participants with oral candidiasis, $13(21.0 \%)$ had clinical diagnosis, where erythematous subtype was present in all cases and pseudomembranous subtype in 12 individuals. Cytopathological analysis revealed more 17 (27.4\%) cases, without oral lesion indicative of candidiasis.

Conclusions: Oral candidiasis is common among patients with hematologic disease, and the cytopathological exam proved to be a useful tool, confirming clinical diagnosis of candidiasis and identifying subclinical cases. These data are of great relevance considering the possible complications that these patients may develop such as longer hospitalizations, worsening of the general condition due to candidemia and even death.

\section{Background}

Hematologic diseases and their treatment may directly or indirectly compromise the oral tissues. ${ }^{1}$ Several systemic diseases have oral manifestations, requiring a thorough clinical and laboratory exams for establishing a definitive diagnosis. ${ }^{2}$ According to the literature, $40 \%$ of oncologic patients receiving chemotherapy present oral complications arising from its toxicity, such as mucositis, infections, salivary gland dysfunction, taste dysfunction, and pain. ${ }^{3,4}$

Candidiasis is an opportunistic yeast infection, and studies suggest that about $40-60 \%$ of the overall population carry oral Candida species, ${ }^{5}$ among which Candida albicans is the most prevalent. ${ }^{6}$ This yeast may cause mild, acute, or chronic alterations, superficial or deep, and of variable clinical presentations. ${ }^{7}$

Candidiasis accounts for $80 \%$ of fungal infections in hospitalized patients. ${ }^{5,8}$ Although fungus may comprise part of the human microbiota, certain local and systemic factors may promote their growth and lead to diseases. ${ }^{9}$ These factors include the use of denture, inhaled or systemic corticosteroids, xerostomia, endocrine diseases, human immunodeficiency virus (HIV), leukemia, malnutrition, immunosuppression, radiotherapy, chemotherapy, and long-term use of antibiotics. ${ }^{9}$ 
Oral candidiasis has a variety of clinical presentations and the diagnosis is often based only in the clinical aspects. ${ }^{7}$ Although pseudomembranous oral candidiasis is readily recognized, the same does not happen for other clinical forms, and it is necessary to perform additional methods to confirm the diagnosis. ${ }^{10}$ Oral cytopathology is a non-invasive, safe, inexpensive, easy to perform, and effective method employed for diagnosing candidiasis..$^{8,11-13}$ Nowadays, cytopathological technique is one of the methods of choice for early diagnosis of candidiasis. ${ }^{11,12}$

Considering that oral candidiasis can be more prevalent in patients with hematologic diseases aggravating their situation, early diagnosis is very important as well as improving its efficacy by the association between physical and cytological exams. The purpose of this study is to verify the prevalence of oral candidiasis in patients with hematologic diseases through clinical and cytopathological exams.

\section{Methods}

This is a cross-sectional and observational study, approved by the Research Ethics Committee of Hospital Universitário Antônio Pedro of the Universidade Federal Fluminense, Niterói-RJ, Brazil (503177158.000.5243), and each subject signed an informed consent form.

The sample consisted of 62 participants hospitalized in the hematology clinic, diagnosed with hematologic diseases (lymphoma, multiple myeloma, leukemia, myelodysplastic syndrome, myeloproliferative syndrome, lymphoproliferative syndrome, hemolytic anemia or anemia, and idiopathic thrombocytopenic purpura), and able to afford the oral mucosa examination. Research participants of both sexes and over 18 years of age were recruited between 2014 and 2016.

Excluding criteria were: individuals with intellectual disability, pregnant and nursing women, and individuals who did not agree with the oral exam or presented insufficient data for analysis.

Information regarding demographic characteristics, habits, medical history, and relevant information about the disease was collected. All patients were submitted to an oral mucosal exam and an oral photographic documentation was obtained by using a Nikon D60 digital camera with a 70-300 mm F/45.6 macro sigma lens and Fc100 circular macro flash. Scraps from oral mucosa were obtained with a sterile cytobrush (Kolplast $\AA_{\text {, Brazil). }}$

The following collected protocol was used: In the absence of oral lesions, smears were collected from the entire oral mucosa for the Papanicolaou staining and periodic acid-Schiff (PAS); In the presence of oral lesion suggestive of candidiasis, a smear was collected from the lesion area for PAS and another smear from the entire oral mucosa (except from the lesion area) for Papanicolaou staining and PAS. The Papanicolaou staining and PAS technique was carried out and cytopathological analysis was performed at the Pathology Service of the Antônio Pedro University Hospital (Universidade Federal Fluminense) by a cytopathologist expert. The diagnosis of oral candidiasis was based on the presence of keratinized 
clustered cells, often overlapped, reflecting marked parakeratosis, pseudo-hyphae and hyphae of Candida $s p^{14}$

Statistical Package for the Social Sciences software (SPSS - version 23.0) was used for statistical analysis. The statistical description of the studied variables was performed by means of proportions (when variables were categorical) and of means, standard deviations, median and minimum-maximum values (when variables were numerical). Pearson's chi-square test and the intraclass correlation of Phi were employed for statistical analysis for two independent samples with equal-variance or unequalvariance. For correlation analysis, besides analysing each form of candidiasis (pseudomembranous, erythematous, and angular cheilitis), we also grouped all forms and considered it as clinic candidiasis. The level of statistical significance used for all analyses was $5 \%(p<0.05)$.

\section{Results}

Of the 62 participants, men (56.5\%) and white skin color (82.3\%) were the most prevalent. The age ranged from 20 to 85 years with a mean of 57 years ( $S D=15$ years). Lymphoma was the most frequent hematologic disease accounting $24.2 \%$ of the cases (Table 1 ). 
Table 1

Demographic and clinical characteristics of 62 participants with hematologic diseases

\begin{tabular}{|ll|}
\hline Data & Participants \\
\hline Gender & - \\
Women (\%) & $27(43.5 \%)$ \\
\hline Skin color & $35(56.5 \%)$ \\
White (\%) & - \\
Non-white (\%) & $51(82.3 \%)$ \\
\hline Age (mean \pm SD, years) & $11(17.3 \%)$ \\
\hline Hematologic Disease & $57 \pm 15$ \\
Lymphoma (\%) & - \\
Leukemia (\%) & $15(24.2 \%)$ \\
Multiple Myeloma (\%) & $10(16.1 \%)$ \\
Myelodysplastic Syndrome (\%) & $10(16.1 \%)$ \\
Myeloproliferative Syndrome (\%) & $10(16.1 \%)$ \\
Idiopathic Thrombocytopenic Purpura (\%) & $4(6.5 \%)$ \\
Hemolytic Anemia (\%) & $4(6.5 \%)$ \\
No conclusive diagnsostic (\%) & $5(8.1 \%)$ \\
\hline
\end{tabular}

Among these individuals, 30 (48.4\%) had diagnosis of oral candidiasis. Of these participants, 13 (21.0\%) had clinical diagnosis, where erythematous subtype was present in all cases and pseudomembranous subtype in 12 (92.3\%) individuals. All of them were confirmed by cytopathological analysis as candidiasis (Figure 1 ).

Cytopathological analysis revealed more $17(27.4 \%)$ cases, without oral lesion indicative of candidiasis (Table 2 and Figure 2). 
Table 2

Prevalence distribution of candidiasis in 62 participants with hematologic diseases, according to the clinical and/or cytopathological diagnosis of candidiasis.

\begin{tabular}{|lll|}
\hline Oral Candidiasis & $\mathbf{N}(\%)$ & Cytopathological Candidiasis \\
\hline Clinical Candidiasis & $13(21.0 \%)$ & $13(43.3 \%)$ \\
\hline Absence of clinical Candidiasis & $49(79.0 \%)$ & $17(56.7 \%)$ \\
\hline Total & $62(100 \%)$ & $30(100 \%)$ \\
\hline
\end{tabular}

By analysing the correlation between cytopathologic diagnosis and oral alterations, we found candidiasis cytopathologic diagnosis to be correlated with: erythematous candidiasis, pseudomembranous candidiasis, clinical candidiasis (when pseudomembranous, erythematous, and angular cheilitis were grouped), hyperplastic lesion, and coated tongue. Angular cheilitis and mucositis could not be analysed because data were not sufficient (Table 3 ). 
Table 3

Results of the correlation analysis between candidiasis cytopathologic diagnosis and oral alterations

\begin{tabular}{|c|c|c|c|c|c|}
\hline \multirow[t]{2}{*}{ Oral Alterations } & \multirow[t]{2}{*}{$\mathbf{N}$} & \multirow[t]{2}{*}{$\%$} & \multirow[t]{2}{*}{$P$} & \multicolumn{2}{|l|}{$95 \%$ IC } \\
\hline & & & & Upper Limit & Lower Limit \\
\hline Lip dryness & 34 & 54.8 & .068 & .101 & .113 \\
\hline Lip ulceration & 9 & 14.5 & .329 & .468 & .487 \\
\hline Labial crust & 4 & 6.5 & .943 & 1.000 & 1.000 \\
\hline Angular cheilitis & 6 & 9.7 & ID & ID & ID \\
\hline Fissured tongue & 18 & 29.0 & .457 & .563 & .583 \\
\hline Coated tongue ${ }^{* *}$ & 17 & 27.4 & .012 & .015 & .020 \\
\hline Atrophic tongue & 14 & 22.6 & .090 & .117 & .130 \\
\hline Hyperpigmentation & 13 & 21.0 & .644 & .740 & .757 \\
\hline Clinical candidiasis $¥$ ** & 12 & 19.4 & .000 & 0.000 & .000 \\
\hline Pseudomembranous candidiasis** & 9 & 14.5 & .000 & 0.000 & .000 \\
\hline Erythematous candidiasis** & 8 & 12.9 & .002 & .001 & .003 \\
\hline Denture stomatitis & 5 & 8.0 & .511 & .701 & .719 \\
\hline Varicosities & 3 & 4.8 & .283 & .349 & .367 \\
\hline Fordyce granules & 1 & 1.61 & .960 & 1.000 & 1.000 \\
\hline Mucositis & 4 & 6.5 & ID & ID & ID \\
\hline Traumatic ulcer & 4 & 6.5 & .943 & 1.000 & 1.000 \\
\hline Hyperplastic lesion** & 3 & 4.8 & .032 & .043 & .051 \\
\hline Hematoma & 1 & 1.61 & .513 & .599 & .618 \\
\hline Morsicatio buccarum & 13 & 21.0 & .296 & .477 & .497 \\
\hline Petechiae or ecchymosis & 2 & 3.2 & .949 & 1.000 & 1.000 \\
\hline Mouth bleeding & 13 & 21.0 & .960 & 1.000 & 1.000 \\
\hline Thick saliva & 26 & 41.9 & .671 & .722 & .740 \\
\hline Oral dryness & 27 & 43.5 & .120 & .170 & .185 \\
\hline Periodontal disease & 2 & 3.2 & .168 & .180 & .195 \\
\hline
\end{tabular}

$\left.{ }^{(\star}\right) \mathrm{p}<0,05$; ( $\left.¥\right)$ could not be analysed because data were not sufficient. 


\begin{tabular}{|lllllll|}
\hline Oral Alterations & $\mathbf{N}$ & $\%$ & $\boldsymbol{P}$ & \multicolumn{2}{l|}{$\mathbf{9 5 \%}$ IC } \\
\cline { 5 - 7 } & & & & & Upper Limit & Lower Limit \\
\hline Herpes simplex & 1 & 1.61 & .960 & 1.000 & 1.000 \\
\hline Mandibular tori & 1 & 1.61 & .296 & .477 & .497 \\
\hline Exostosis & 1 & 1.61 & .296 & .477 & .497 \\
\hline$(* *) p<0,05 ;(¥)$ could not be analysed because data were not sufficient. & \\
\hline
\end{tabular}

\section{Discussion}

Malignant neoplasms are one of the main causes of death and morbidity, representing a challenge for the health system in the treatment of patients. ${ }^{15}$ According to data from the National Institute of Cancer (INCA), ${ }^{16}$ about 680 thousand new cases of cancer were diagnosed in 2020. Hematologic malignancies are among the ten most common types of neoplasms in Brazil, deserving attention due to its high mortality rate (2-3\%). In our study, most patients presented leukemia and lymphoma, corroborating results reported in the literature, which state that leukemia and lymphoma are the most common oncohematological diseases. ${ }^{17}$

Smith et al. ${ }^{18}$ conducted a study with hematologic patients and found $68 \%$ of them to be men with mean age of 70 years. However, Sabater Recolons et al. ${ }^{19}$ found a mild predominance in women (51\%) with mean age of 48 years. Schelenz et al. ${ }^{20}$ verified a slight predominance among females (53\%) with mean age of 61 years (18 to 90 years). The literature shows a higher incidence in older individuals and a predilection for men regarding to multiple myeloma, lymphomas, and leukemias. ${ }^{4}$ We found most of the hematologic diseases to be lymphomas, leukemias, and multiple myeloma, which may explain the slight predominance among males (56.5\%) with mean age of 57 years.

According to the literature, most of oncologic patients receiving chemotherapy present oral complications arising from its toxicity, such as opportunistic infection. ${ }^{21,22}$ Candidiasis is an opportunistic infection caused by fungi of the genus Candida (Candida spp.) with several clinical manifestations. It is the most common mycosis in the oral cavity, found as a commensal organism in over a half of the world population. ${ }^{23,24}$ Yeasts of the genus Candida are the main cause of nosocomial infection, and represent a challenge for the survival of patients with severe diseases. ${ }^{25}$ Candida spp. is responsible for candidemia - the fourth most common cause of nosocomial infections, - significantly increasing morbidity and mortality rates, and incurring high costs to health institutions. ${ }^{26-28}$

Patients with onco-hematologic diseases and bone marrow transplant have a worse prognosis when they have candidiasis. ${ }^{18,20,29}$ Studies approaching oral candidiasis and hematologic patients, on whom intervention is more difficult, have some limitations. The different investigation methods employed for candidiasis diagnosis make difficult the results comparison. Ponces and Torres et al. ${ }^{30}$ studied leukemic 
children receiving chemotherapy and found $6 \%$ of them with oral candidiasis by clinical diagnosis. Alberth et al. ${ }^{31}$ used culture for candidiasis diagnosis in children with hematologic diseases and found $84 \%$ of them to be positive for the disease.

The present study was the first to analyse the presence of oral candidiasis in onco-hematological patients by cytopathology, and our findings show a high prevalence of oral candidiasis. Previously the prevalence of Candida species had been shown using cultures from the oral cavity without evaluating the presence of oral lesions. Positive culture in the absence of other signs and symptoms does not necessarily imply the presence of the disease. Both physical exam and culture have limitations in the diagnosis of candidiasis. ${ }^{23,25}$ Physical exam may not identify areas with small alterations suggestive of candidiasis, or even misinterpret some erythematous alterations as candidiasis, such as in denture stomatitis unrelated to candidiasis, and mucosa atrophy, which may be associated to anemia or erythroplasia and consequently to oral squamous cell carcinoma. Oral cytopathology is a non-invasive, fast and easy to perform technique that may be used to both confirm the clinical diagnosis of candidiasis and identify lesions that are not detected during inspection or do not have clinical manifestations. ${ }^{14}$ In this study, oral candidiasis was detected in 30 (48.4\%) patients, where cytopathology demonstrated $43.3 \%$ of positive clinical diagnosis of candidiasis. This result may be explained by the fact that the oral manifestations of erythematous and pseudomembranous candidiasis in patients with onco-hematologic diseases are more expressive features, which increase the accuracy of clinical diagnosis. Cytopathology analysis also demonstrated false negative clinical diagnosis. In $56.7 \%$ (17 cases) candidiasis was only identified by cytopathology. It is important to mention that in all these cases the cytopathological aspects correspond to the classical aspects, including the presence of hyphae of Candida associated with inflammatory changes, which differs from colonization of the mucosa. ${ }^{14}$ We considered these cases as subclinical candidiasis - early candidiasis that may represent a risk for patients' prognosis despite the lack of clinical expression, considering the severity of the systemic disease. Thus, cytopathologic exam was an efficient diagnostic tool and well accepted by the participants. Besides confirming the suspected cases of candidiasis, this method also identified the disease in cases which no signs of candidiasis were identified. Although health professionals may encounter some difficulties in exam methods for hospitalized patients with systemic diseases, cytopathologic exam seems to be a reliable method, and we recommend it as part of the protocol for those patients.

We found an association between the cytopathologic diagnosis of candidiasis and different patterns of clinical candidiasis, which was already expected due to the diagnostic criteria. The clinical diagnosis of coated tongue and the cytopathologic diagnosis of candidiasis were also correlated. Some authors consider that coated tongue may be associated with candidiasis, ${ }^{32}$ which was verified in our study. We observed a correlation between hyperplastic lesions and cytopathologic diagnosis, which were considered part of subclinical candidiasis without etiological association between Candida and hyperplastic lesions. Reports on denture stomatitis are quite controversial: some authors consider it as a form of candidiasis while others consider that lesions may be infected by Candida spp. ${ }^{14,32}$ In our study, denture stomatitis was not correlated with the cytopathologic diagnosis of candidiasis. We believe 
denture stomatitis to be an inflammatory response associated with poor hygiene, long-term prosthesis use, and a hypersensitivity response to the polymer. Candidiasis may or not be present in those cases, and the poor hygiene and prostheses roughness may contribute to microorganisms' proliferation and disease progression.

\section{Conclusion}

Oral candidiasis is common among onco-hematologic patients. Cytopathologic exam identified more cases of candidiasis than physical exam, and demonstrated to be a useful diagnostic tool, easy to perform, painless, low-cost, and well accepted by patients. We found cytopathology to be important in the diagnosis of candidiasis, confirming clinical diagnosis and identifying subclinical cases.

\section{Abbreviations}

PAS: Periodic acid-Schiff.

\section{Declarations}

Ethics approval and consent to participate: This work was approved by the Research Ethics Committee of Hospital Universitário Antônio Pedro of the Universidade Federal Fluminense (\#503177158.000.5243), and was conducted in full accordance with Declaration of Helsinki. The participant provided an informed consent declaration to permit the use of images and medical information and the manuscript is in accordance with the Institutional Ethics Committee.

Consent for publication: Not applicable.

Availability of data and materials: Not applicable.

Competing interests: The authors declare that there is no conflict of interest.

Funding: This research received no specific grant from funding agencies in the public, commercial, or notfor-profit sectors.

Authors' contributions: FGAP conceptualized and designed the study, evaluated cytopathological glass slides, collected clinical, and sociodemographic data, interpreted results, and wrote the manuscript. AM conceptualized and designed the study, and evaluated cytopathological glass slides. JTW, LCM and BLSP interpreted results, and wrote the manuscript. ASJ conceptualized and designed the study and wrote the manuscript.

Acknowledgements: Not applicable.

\section{References}


1. Adeyemo TA, Adeyemo WL, Adediran A, Akinbami AJA, Akanmu AS. Orofacial manifestations of hematological disorders: anemia and hemostatic disorders. Indian J Dent Res. 2011;22(3):454-461. doi:10.4103/0970-9290.87070

2. Napeñas JJ, Brennan MT, Elad S. Oral Manifestations of Systemic Diseases. Dermatol Clin. 2020;38(4):495-505. doi:10.1016/j.det.2020.05.010

3. Hespanhol FL, Tinoco EMB, Teixeira HG de C, Falabella MEV, Assis NM de SP. Manifestações bucais em pacientes submetidos à quimioterapia. Ciênc saúde coletiva. 2010;15:1085-1094. doi:10.1590/S1413-81232010000700016

4. Oliveira KKV de, Dias I de O, Martins ICV, Oliveira IF, Valadão AF, Motta PG da. Manifestações orais nas doenças hematológicas: revisão de literatura DOI: http://dx.doi.org/10.5892/ruvrd.v13i1. 2275. Revista da Universidade Vale do Rio Verde. 2015;13(2):216-235. doi:10.5892/ruvrd.v13i2.2275

5. Leonardo S Barbedo1, \& Diana BG Sgarbi2. Candidíase. Accessed April 18, 2020. http://ole.uff.br/wpcontent/uploads/sites/303/2018/02/r22-1-2010-4-Candidiase.pdf

6. Gharibpour F, Shirban F, Bagherniya M, Nosouhian M, Sathyapalan T, Sahebkar A. The Effects of Nutraceuticals and Herbal Medicine on Candida albicans in Oral Candidiasis: A Comprehensive Review. Adv Exp Med Biol. 2021;1308:225-248. doi:10.1007/978-3-030-64872-5_16

7. Hellstein JW, Marek CL. Candidiasis: Red and White Manifestations in the Oral Cavity. Head Neck Pathol. 2019;13(1):25-32. doi:10.1007/s12105-019-01004-6

8. Siqueira J da SS, Batista SA, Silva Jr. A, Ferreira MF, Agostini M, Torres SR. Candidíase oral em pacientes internados em UTI. Revista Brasileira de Odontologia. 2014;71(2):176-179.

9. Cho E, Park Y, Kim K-Y, et al. Clinical Characteristics and Relevance of Oral Candida Biofilm in Tongue Smears. Journal of Fungi. 2021;7(2):77. doi:10.3390/jof7020077

10. Dias EP, Sayed Picciani BL, de Carla Batista Santos V, Oliveira Silva-Junior G, Heffer Cantisano M, Silva-Junior A. The advantages of oral cytopathology in the early diagnosis of HIV/AIDS: three case reports. Acta Cytol. 2012;56(4):453-456. doi:10.1159/000335063

11. Braz-Silva PH, Magalhães MHCG, Hofman V, et al. Usefulness of oral cytopathology in the diagnosis of infectious diseases. Cytopathology. 2010;21(5):285-299. doi:10.1111/j.1365-2303.2010.00779.x

12. Verma R, Singh A, Badni M, Chandra A, Gupta S, Verma R. Evaluation of exfoliative cytology in the diagnosis of oral premalignant and malignant lesions: A cytomorphometric analysis. Dent Res $J$ (Isfahan). 2015;12(1):83-88. doi:10.4103/1735-3327.150339

13. Fontes KBF da C, Cunha KSG, Rodrigues FR, Silva LE da, Dias EP. Concordance between cytopathology and incisional biopsy in the diagnosis of oral squamous cell carcinoma. Braz Oral Res. 
14. Picciani BLS, Michalski-Santos B, Carneiro S, et al. Oral candidiasis in patients with psoriasis: correlation of oral examination and cytopathological evaluation with psoriasis disease severity and treatment. J Am Acad Dermatol. 2013;68(6):986-991. doi:10.1016/j.jaad.2012.11.033

15. Siegel RL, Miller KD, Jemal A. Cancer statistics, 2019. CA Cancer J Clin. 2019;69(1):7-34. doi:10.3322/caac. 21551

16. INCA - Instituto Nacional de Câncer. INCA - Instituto Nacional de Câncer. Accessed October 18, 2021. https://www.inca.gov.br/

17. Dou H-J, Zhu H. [Clinical analysis of 500 cases of hematological diseases with oral presentation as the first manifestation]. Shanghai Kou Qiang Yi Xue. 2020;29(6):638-641.

18. Smith A, Howell D, Patmore R, Jack A, Roman E. Incidence of haematological malignancy by sub-type: a report from the Haematological Malignancy Research Network. Br J Cancer. 2011;105(11):1684-1692. doi:10.1038/bjc.2011.450

19. Sabater Recolons M del M, López JL, Campillo MER de R, Küstner EC, Vidal JMC. Buccodental health and oral mucositis. Clinical study in patients with hematological diseases. Med Oral Patol Oral Cir Bucal. 2006;11:497-502.

20. Schelenz S, Abdallah S, Gray G, et al. Epidemiology of oral yeast colonization and infection in patients with hematological malignancies, head neck and solid tumors. J Oral Pathol Med. 2011;40(1):83-89. doi:10.1111/j.1600-0714.2010.00937.x

21. Ohmoto A, Fuji S. Infection profiles of different chemotherapy regimens and the clinical feasibility of antimicrobial prophylaxis in patients with DLBCL. Blood Rev. 2021;46:100738. doi:10.1016/j.blre.2020.100738

22. Atkins S, He F. Chemotherapy and Beyond: Infections in the Era of Old and New Treatments for Hematologic Malignancies. Infect Dis Clin North Am. 2019;33(2):289-309. doi:10.1016/j.idc.2019.01.001

23. Coronado-Castellote L, Jiménez-Soriano Y. Clinical and microbiological diagnosis of oral candidiasis. J Clin Exp Dent. 2013;5(5):e279-e286. doi:10.4317/jced.51242

24. Shao T-Y, Ang WXG, Jiang TT, et al. Commensal Candida albicans Positively Calibrates Systemic Th17 Immunological Responses. Cell Host Microbe. 2019;25(3):404-417.e6. doi:10.1016/j.chom.2019.02.004

25. Kao AS, Brandt ME, Pruitt WR, et al. The epidemiology of candidemia in two United States cities: results of a population-based active surveillance. Clin Infect Dis. 1999;29(5):1164-1170. doi:10.1086/313450 
26. Kauffman CA. Fungal Infections. Proc Am Thorac Soc. 2006;3(1):35-40. doi:10.1513/pats.200510110JH

27. Latgé J-P, Calderone R. Host-microbe interactions: fungi invasive human fungal opportunistic infections. Current Opinion in Microbiology. 2002;5(4):355-358. doi:10.1016/S1369-5274(02)00343-0

28. Wisplinghoff H, Ebbers $J$, Geurtz L, et al. Nosocomial bloodstream infections due to Candida spp. in the USA: species distribution, clinical features and antifungal susceptibilities. Int J Antimicrob Agents. 2014;43(1):78-81. doi:10.1016/j.ijantimicag.2013.09.005

29. Edmond MB, Wallace SE, McClish DK, Pfaller MA, Jones RN, Wenzel RP. Nosocomial bloodstream infections in United States hospitals: a three-year analysis. Clin Infect Dis. 1999;29(2):239-244. doi:10.1086/520192

30. Ponce-Torres E, Ruíz-Rodríguez M del S, Alejo-González F, Hernández-Sierra JF, Pozos-Guillén A de J. Oral manifestations in pediatric patients receiving chemotherapy for acute lymphoblastic leukemia. J Clin Pediatr Dent. 2010;34(3):275-279. doi:10.17796/jcpd.34.3.y060151580h301t7

31. Alberth M, Majoros L, Kovalecz G, et al. Significance of oral Candida infections in children with cancer. Pathol Oncol Res. 2006;12(4):237-241. doi:10.1007/BF02893420

32. Gopal S, Wood WA, Lee SJ, et al. Meeting the challenge of hematologic malignancies in sub-Saharan Africa. Blood. 2012;119(22):5078-5087. doi:10.1182/blood-2012-02-387092

\section{Figures}

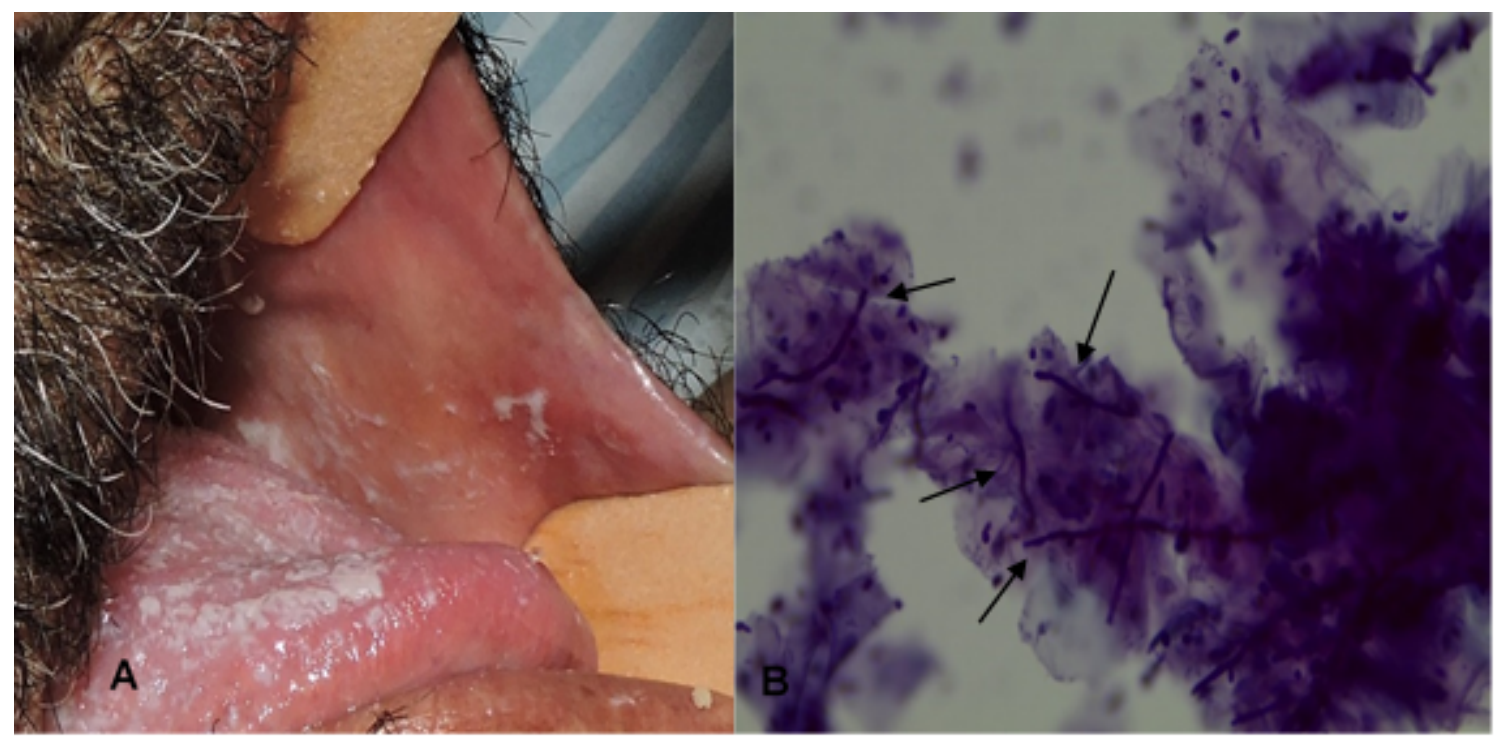

\section{Figure 1}


Participant with clinical candidiasis and photomicrography of oral smear: A. Clinical pseudomembranous candidiasis as white lesions on the tongue and buccal mucosa. B. Photomicrography of smears from the lesion, showing the presence of hyphae (arrows), diagnosed as candidiasis (PAS - 40X).

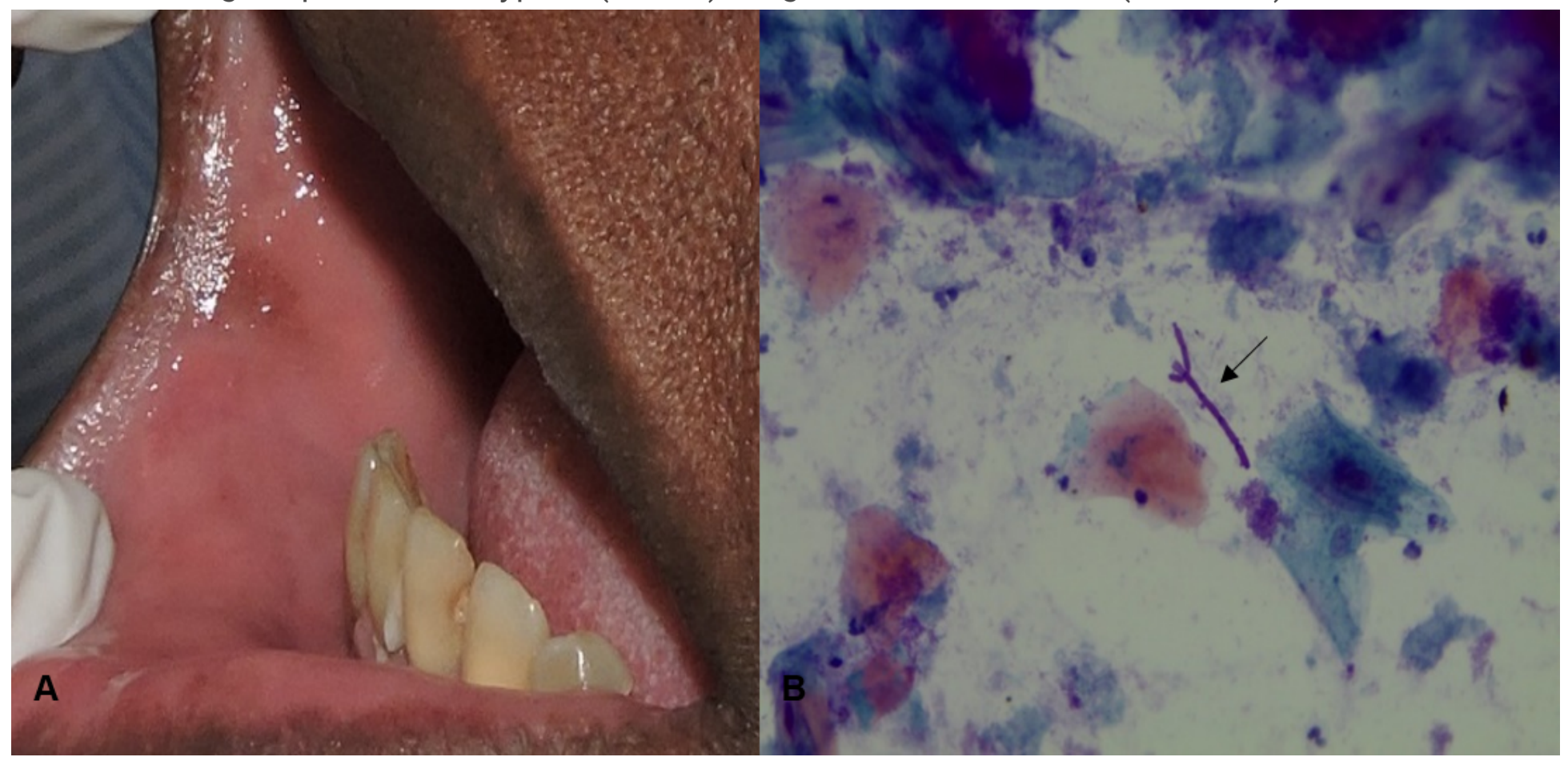

\section{Figure 2}

Participant with normal oral mucosa and photomicrography of oral smear: A. Clinical alteration suggestive of hyperpigmentation due to brown colour. B. Photomicrography of the smear from the oral mucosa showing the presence of hyphae (arrow), diagnosed as candidiasis (PAPA/PAS - 40X). 